\title{
Analyse De L'efficacité Technique Des Exploitations Agricoles Familiales À Maurice
}

\author{
Malick Ndiaye \\ Ecole doctorale: Sciences de l'Homme et de la Société (SHS), \\ Laboratoire de Recherches en Economie de Saint-Louis (LARES), \\ Université Gaston Berger, Saint-Louis, Sénégal
}

Doi: 10.19044/esj.2018.v14n9p143 URL:http://dx.doi.org/10.19044/esj.2018.v14n9p143

\begin{abstract}
This study was undertaken to determine the technical efficiency of family farms and to identify the factors which determine their productivity. All in all, 200 farmers were randomly selected and interviewed using a structured questionnaire to obtain data on agricultural production and input use during 2014. The Data Envelopment Analysis (DEA) technique was used to determine the levels of technical efficiency of such enterprises. Besides, a Tobit regression model was applied to identify the factors influencing the technical efficiency of farmers in agricultural production. The average technical efficiency under the variable scale yield is 0.726 . This implies that it is possible to increase agricultural production by $27.4 \%$ compared to the current level of inputs. The results show that $46.5 \%$ of the sample is technically efficient under variable scale yield. The results showed that productivity is significantly influenced by the sex of the farmer, the area of cultivated land and the wage of labor. It is therefore recommended to undertake a policy to improve the formal education of producers, to build capacity of their organizations and facilitate to them access to land and inputs.
\end{abstract}

Keywords: Family farms, Data Envelopment Analysis (DEA), Tobit regression, Mauritius

\section{Résumé}

La présente étude est entreprise pour déterminer l'efficacité technique des exploitations agricoles familiales et identifier les facteurs qui déterminent leur productivité. Au total, 200 agriculteurs sont sélectionnés au hasard et interrogés à l'aide d'un questionnaire structuré pour obtenir des données relatives à la production agricole, à l'utilisation des intrants au cours de l'année 2014. La technique d'Analyse par Enveloppement de Données (DEA) est utilisée pour déterminer les niveaux d'efficacité technique de ces 
exploitations. En outre, un modèle de régression Tobit est appliqué pour identifier les facteurs influençant l'efficacité technique des exploitations dans la production agricole. L'efficacité technique moyenne dans le cadre du rendement d'échelle variable est de 0,726. Cela implique qu'il est possible d'augmenter la production agricole de $27,4 \%$ par rapport au niveau actuel des intrants. Les résultats montrent que 46,5\% de l'échantillon sont techniquement efficaces sous rendement d'échelle variable. Il en résulte aussi que la productivité est significativement influencée par le sexe du chef d'exploitation, la superficie cultivée et le salaire. Il est donc recommandé d'entreprendre une politique d'amélioration de l'éducation formelle des producteurs, de renforcement des capacités de leurs organisations et d'accès aux terres et aux intrants.

Mots clés : Agriculture familiale, Analyse par Enveloppement de Données (DEA), régression Tobit, Maurice

\section{Introduction}

L'agriculture a été historiquement l'un des piliers de l'économie Mauricienne (IEDOM, 2009) dominée par la culture de la canne à sucre avec environ $90 \%{ }^{1}$ des terres cultivées (UNFCCC, 2010). Actuellement, 1'agriculture Mauricienne se diversifie plus avec la production de spéculations comme: les cultures vivrières, le tabac et le thé (MAIF, 2008). Elles sont cultivées sur $10 \%$ de la superficie totale par quelque 12000 petits producteurs (UNFCCC, 2010). L'Ile Maurice, comme beaucoup de pays en développement, est confronté à des difficultés en intrants de base et des problèmes de la sécurité alimentaire avec la récente explosion des prix alimentaires. Ces problèmes devraient persister en raison de certains changements structurels dans le système alimentaire mondial (Statistics Mauritius, 2014). La production annuelle de cultures vivrières tourne autour de $25 \%$ des besoins alimentaires totaux tandis que les $75 \%$ restants comprenant des aliments de base tels que le blé, le riz, l'oignon, l'ail, la tomate en conserve pour la cuisine et les fruits sont importés (MAIFS, 2013). L'agriculture emploie environ $8,7 \%$ de la population (HRDC, 2012), mais il a augmenté de 0,9\%, passant de 44900 en 2014 à 45300 en 2015 (Statistics Mauritius, 2016). En outre, sa contribution au produit intérieur brut (PIB) du pays a diminué au fil des ans : 3,5\% en 2012, 3,3\% en 2013, 3,7\% en 2014 et 3,5\% en 2015 (Statistics Mauritius, 2014) ; (Statistics Mauritius, 2016). En plus, IFAD, (2013) estime aussi que 8,7\% des Mauriciens vivent dans la pauvreté.

${ }^{1}$ Environ $70 \%$ du secteur de la canne à sucre (43 000 ha) sous gestion d'entreprise (31 unités) et les 23000 ha restants appartenant à environ 23500 personnes (UNFCCC, 2010). 
Dans ces contextes, il est primordial de promouvoir une agriculture efficace, c'est-à-dire, utiliser moins de ressources pour produire davantage. Cela a motivé beaucoup de recherches ces dernières années sur l'ampleur et les sources à l'origine des écarts d'efficacité dans l'agriculture paysanne (Alene et al. 2006). La mesure de l'efficacité a été un domaine de recherche populaire depuis la publication de son article en 1957. Farrell (1957) a développé le concept d'efficacité technique basé sur les relations entre les intrants et les extrants. Depuis, de nombreuses recherches se sont concentrées sur les facteurs explicatifs de l'efficacité technique des ménages agricoles : Chavas et al. (2005) en Gambie ; (Abatania, Hailu, \& Mugera, 2012)au nord du Ghana ; Dhehibi et al. (2014) dans les deux gouvernorats de la Palestine. Ces auteurs trouvent que le genre, l'âge, le niveau d'éducation du chef de ménage, son expérience, la main-d'œuvre, l'accès au crédit et aux services de vulgarisation, et l'appartenance à une coopérative affectent de manière significative l'efficacité technique. Un certain nombre d'études ont également été menées sur l'efficacité technique de la production de certaines filières agricoles : Coelli et al. (2002) pour la production du riz au Bangladesh ; Rios et Shively (2005) pour la production de café au Vietnam ; Padilla-Fernandez et Nuthall (2009) pour la production de canne à sucre au Philippines; Chiona (2011) pour la production de maïs en Zambie et Clemente et al. (2015) pour production d'agrumes au Brésil. D'où l'intérêt de cet article qui aborde la problématique de l'analyse de l'efficacité des exploitations et de la production agricole Mauricienne. Cette analyse est importante dans le contexte de la promotion des programmes et projets de développement agricole et de la contribution de l'agriculture à l'économie nationale Mauricienne.

Le but de cette étude est d'étudier l'efficacité technique des exploitations agricoles familiales à Maurice. Ainsi les questions spécifiques posées par cette étude sont: Quel est le niveau d'efficacité technique des facteurs de production ? Quels sont les facteurs socio-économiques qui influencent l'efficacité technique des exploitations agricoles ?

Ceci est réalisé en estimant leur efficacité technique et en établissant les facteurs qui influencent celle-ci. Cette efficacité est estimée dans un cadre d'analyse d'enveloppe de données (DEA) pour différencier les agriculteurs efficaces des agriculteurs inefficaces.

\section{Littérature empirique sur l'efficacité technique des systèmes de production agricoles}

Diverses études se sont intéressées sur l'efficacité technique en utilisant la méthode DEA (Data Envelopment Analysis) (Javed et al., 2010 ; Heidari, et al., 2011 ; Bhatt et Bhat, 2014) ou la méthode de frontière stochastique (Stochastic frontier models) (Rahman et Umar, 2009; Dhehibi, et al., 2014). Elles admettent les uns après les autres que l'âge, le sexe, 
l'expérience, l'appartenance à une organisation, la main d'œuvre, accès aux crédits, et la taille de l'exploitation et la superficie cultivée sont les facteurs socioéconomiques qui déterminent l'efficacité techniques des producteurs (Chavas et al.,2005 ; Abatania et al., 2012 ; Dhehibi et al., 2014). En effet, Chavas et al. (2005) a étudié l'efficacité des ménages agricoles en Gambie en utilisant des mesures non paramétriques. Ces derniers ont utilisé une analyse économétrique basée sur le modèle Tobit pour identifier les facteurs explicatifs de l'efficacité technique de production. Ces auteurs trouvent que les scores d'efficacité des ménages varient de 0,08 à 0,95 et que $85 \%$ des ménages sont techniquement efficaces. Ils expliquent la présence d'inefficacité par les mauvaises capacités de gestion des ménages, l'inefficience du marché du travail et du crédit et la mauvaise organisation des ménages. Il résulte de leur travail que le genre de ménage, le statut de pauvreté et l'insécurité alimentaire exercent une influence significative sur l'efficacité.

Abatania et al. (2012) ont étudié l'efficacité technique de 189 exploitations agricoles dans le nord du Ghana en utilisant la méthode DEA. Ils trouvent un score d'efficacité technique moyen de 0,77. Ce qui implique que, en moyenne, les exploitations agricoles pourraient réduire leurs intrants agricoles de $22,74 \%$ tout en produisant le niveau de production actuel. Ils montrent que le sexe et l'âge du chef de ménage, la main-d'œuvre salariée, la localisation géographique des exploitations affectent de manière significative l'efficacité technique.

S'inscrivent dans cette logique les travaux de Dhehibi et al. (2014). Ces auteurs ont analysé l'efficacité technique et les déterminants de la production agricole des exploitations en Palestine. Une frontière de production stochastique de Cobb-Douglas a été utilisée portant sur un échantillon de 100 exploitations pluviales. Il en résulte qu'en moyenne que les exploitations de l'échantillon peuvent potentiellement augmenter leur productivité de $28 \%$ grâce à une utilisation plus efficace des intrants. Ils estiment que le niveau d'éducation des agriculteurs, leur expérience, l'accès au crédit et aux services de vulgarisation, et l'appartenance à une coopérative sont les principaux déterminants de l'efficacité technique. Une étude similaire et récente a été faite au Bénin où une efficacité technique de 0,95 pour le maïs et 0,99 pour le coton a été déterminé dans des exploitations familiales d'agro-éleveurs peuls (Diogo, et al., 2017). Ils trouvent que, d'une part, la taille du cheptel bovin et la superficie emblavée ont influencé significativement le parcage et d'autre part que, la main d'œuvre a influencé significativement l'application de l'engrais minéral.

En outre, Bhatt et Bhat (2014) ont étudié le lien entre la taille des exploitations et l'efficacité technique des producteurs dans le district Pulwama de Jammu-et-Cachemire en Inde. L'approche non paramétrique a été choisie. La méthode DEA a été utilisée pour évaluer les scores d'efficacité 
technique de 461 producteurs enquêtés. L'étude montre que les grandes exploitations tendent à avoir un revenu agricole net plus élevé par acre et sont techniquement efficaces comparativement aux autres catégories de petites exploitations. Ils constatent que l'occupation, l'expérience, l'adhésion à une organisation du chef de ménage, la taille du ménage, la superficie cultivée, et le type de semence sont des déterminants importants qui influencent les écarts entre la taille des exploitations et l'efficacité technique des producteurs.

D’autres études empiriques ont été menées sur la production agricole de certaines cultures pour quantifier exactement le niveau d'efficacité atteint par les producteurs dans plusieurs pays.

Dans ce sens, les déterminants de l'efficacité technique de la production végétale ont étudié par Rahman et Umar (2009) dans la zone de Lafia de l'état de Nasarawa au Nigeria. Ils trouvent que l'efficacité technique de la production agricole varie de 0,32 à 0,89 avec une moyenne de 0,69. Ils estiment aussi que l'âge, le sexe, l'état matrimonial, la taille du ménage, d'autres professions et la propriété foncière sont les déterminants significatifs qui expliquent les variations observées dans l'efficacité technique.

Au Bangladesh, Coelli et al. (2002) ont mesuré l'efficacité technique, allocative et à l'échelle de la culture du riz. Ils estiment des scores d'efficacités technique, allocative, de coût et de production de riz à la saison sèche de 0,$694 ; 0,813 ; 0,562$ et 0,949 respectivement. Ils affirment aussi que les familles nombreuses sont susceptibles d'être plus inefficaces que les agriculteurs qui ont un meilleur accès aux marchés d'intrants. Par contre, les agriculteurs qui font moins de travail agricole ont tendance à être plus efficaces.

L'efficacité technique du système riz-blé a aussi été étudiée par Javed et al. (2010) à Punjab au Pakistan. Appliquant les modèles d'analyses DEA et Tobit, ces auteurs trouvent que l'efficacité technique est de $32 \%$ en moyenne et que les intrants pourraient être réduits de $17 \%$ sans réduire le niveau de production avec la technologie existante. Il résulte de leur étude que la taille des exploitations, l'âge des exploitants agricoles et les distances des marchés l'affectent positivement l'efficacité technique des producteurs.

Les mesures de taille et d'efficacité de l'exploitation pour le café au Vietnam ont été étudiées par Rios et Shively (2005) en utilisant une approche en deux étapes : un modèle DEA suivi d'une analyse de régression. Selon eux, les grandes exploitations sont plus efficaces sur le plan technique et économique que les petites exploitations. En outre, la longueur du tuyau d'irrigation et l'enseignement supérieur réduisent l'efficacité dans les petites exploitations.

Padilla-Fernandez et Nuthall (2009) trouvent que le score d'efficacité technique moyen est de 0,78 dans les exploitations de canne à sucre au Central Negros Area, Philippines. Ce qui implique que les $22 \%$ de marges 
d'amélioration expliquent les pertes enregistrées dans leurs exploitations. Il ressort de leurs résultats que les différences d'utilisation des intrants entre les exploitations techniquement efficaces et ceux techniquement inefficaces étaient très importants en termes de superficie, de semences et d'intrants de main-d'œuvre.

Dans cette même logique, les niveaux d'efficacité technique et allocative des petits producteurs de maïs en Zambie ont été estimés par Chiona (2011) en appliquant l'approche DEA et une équation de régression MCO. Les résultats montrent que seulement $15 \%$ de l'échantillon sont techniquement efficace et seulement $12 \%$ sont entièrement efficaces en fonction de l'allocation. Il détermine que, les méthodes de labour mécanisé, l'utilisation d'engrais et de semences certifiées, l'éducation formelle des chefs de ménage et la participation à des activités agricoles sont corrélées positivement avec l'efficacité technique. La même approche méthodologique a été appliquée par Clemente et al. (2015) pour analyser efficacité technique des producteurs d'agrumes en 2009 et 2010 dans l'état de Sao Paulo au Brésil. Cependant, ils estiment que la «formation» et l'expérience» sont les variables qui contribuent le plus à accroître l'efficacité technique des producteurs de cette zone. C'est avec cette même méthodologie que Yusuf et Malomo (2009) trouvent des scores d'efficacité différents entre les grandes exploitations $(0,89)$, les exploitations moyennes $(0,87)$ et les petites exploitations $(0,86)$ de volaille dans l'État d'Ogun au Nigéria. Selon ces auteurs, les années d'expérience agricole ont eu une incidence positive sur l'efficacité, tandis que la taille du ménage a eu une efficacité négative. Egalement, Heidari et al. (2011) ont déterminé l'efficacité économique de l'utilisation des ressources dans les fermes de production de poulets de chair. Les valeurs moyennes des rendements techniques et des rendements des agriculteurs ont été de 0,92 et 0,93 respectivement. Ils affirment qu'environ $10 \%$ du total des ressources d'intrants pourraient être économisés si les agriculteurs utilisent leurs intrants de manière efficiente.

Par conséquent nous affirmons avec Osawe et al., (2008) que les agriculteurs doivent donc utiliser les ressources disponibles de manière intensive et rationnelle afin de produire un meilleur rendement et d'être plus efficaces techniquement.

\section{Méthodologie \\ Zone d'étude}

La République de Maurice est un pays insulaire dans l'océan Indien, à $950 \mathrm{~km}$ à l'Est de Madagascar. Elle couvre une superficie totale de $2040 \mathrm{~km}^{2}$ et est constituée de l'île Maurice (communément appelé Maurice) elle-même et les îles de Rodrigues, Agalega et de l'archipel des Chagos. 
Maurice couvre une superficie de $1865 \mathrm{~km}^{2}$ et compte une population estimée à 1250000 habitants en 2014 avec un taux de croissance annuelle de 0,4\% (USAID, 2015). Maurice est constitué de quatre (4) zones agroécologiques (Est, Ouest, Nord et Sud) et administrativement subdivisé en neuf (9) districts (régions). Le Nord comprend les régions de Port Louis, Pamplemousses et Riviere du Rempart, le Sud aligne Grand Port et Savanne, l'Est englobe Moka, Flacq, et enfin 1'Ouest qui est occupé par Black River et les Plaines Wilhems (Figure 1).

Son climat comprend deux saisons : la saison d'été chaude et pluvieuse (Novembre-Avril) et la saison d'hiver est fraîche et relativement sèche entre Mai et Octobre (Mardamootoo, 2009). La température annuelle moyenne de l'air est $22^{\circ} \mathrm{C}$ et la moyenne annuelle des précipitations de Maurice est de 2 $041 \mathrm{~mm}$ (Le Roux, 2005). Les variations de températures et de précipitations d'une région à l'autre permettent les subdivisions de l'île en zones subhumide, humide et super humide. La zone subhumide est limitée à basse altitude sur la côte ouest et les plaines du nord où la pluviométrie totale est inférieure à 1250 $\mathrm{mm}$. La région humide se produit à des altitudes intermédiaires sur les côtes occidentales et septentrionales avec des précipitations entre $1250 \mathrm{~mm}$ et 2000 $\mathrm{mm}$, et sur les plaines de la côte orientale, où la pluviométrie est inférieure à $2500 \mathrm{~mm}$. La région super humide situe au-dessus de $450 \mathrm{~m}$ sur la côte ouest et au-dessus de $400 \mathrm{~m}$ sur la côte Est, où les précipitations dépassent 2000 $\mathrm{mm}$.

Les sols sont presque exclusivement développés à partir de roches volcaniques basiques, principalement des laves basaltiques olivine, d'âges très différents (Mardamootoo, 2009). Les sols importants pour l'agriculture de cette région sont subdivisés en deux groupes principaux : les sols maturité ou latosols ferrallitiques et les sols latosolic immatures (Kamminga, 2008).

Les ressources en eau renouvelables sont estimées à $2,751 \mathrm{~km}^{3} /$ an et celui des ressources en eau exploitables sont estimées à $1,083 \mathrm{~km}^{3} /$ an. Le retrait total de l'eau est estimé à 725 millions de m³/an (2003), dont 491214 $\mathrm{m}^{3}$ sont destinés à l'agriculture (FAO, 2005).

L'agriculture occupe 9\% dans l'agriculture de la population active (USAID, 2015) et sa part dans le PIB est faible (3,5\% en 2015) (Statistics Mauritius, 2016). Les superficies cultivées sont de 106000 ha, soit 52\% de la superficie totale de Maurice, dont les terres arables couvrent 100000 ha et les cultures permanentes 6000 ha (UNFCCC, 2010). La zone dispose d'écosystèmes relativement variés lui conférant des potentialités agricoles importants. L'essentiel de la production agricole concerne : la canne à sucre, le thé, le tabac et les cultures vivrières qui comprend : les haricots et pois, les patates, le maïs, l'arachide, l'oignon la tomate et les fruits et légumes.

La région est très propice à l'agriculture, mais le développement de cette dernière a été entravé par le climat sec et le manque d'installations 
d'irrigation. L'extension du réseau d'irrigation permet non seulement de booster la productivité mais aussi de stabiliser la production et rend les activités agricoles moins risqué notamment avec le changement climatique.

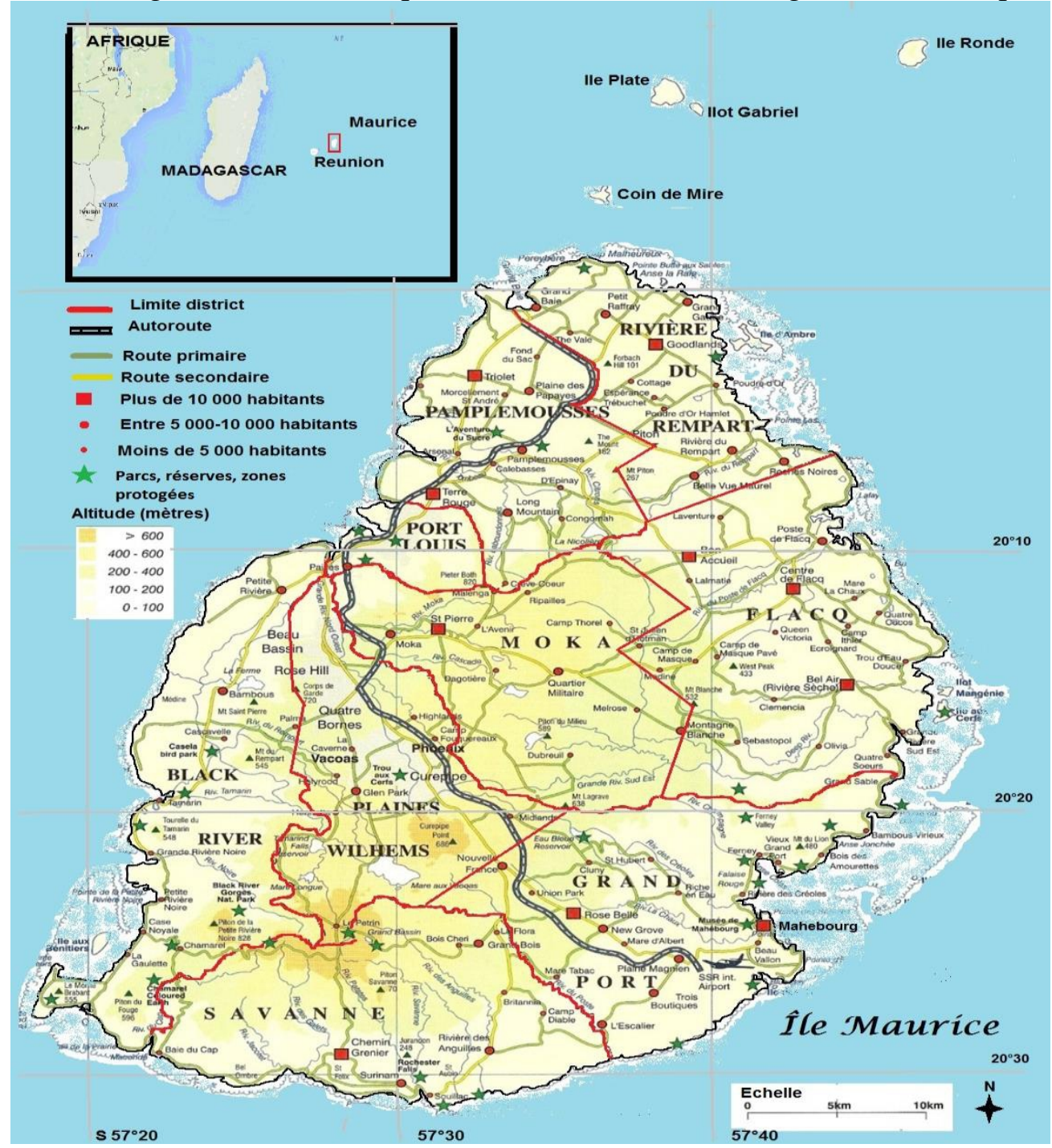

Figure 1 : Présentation géographique de Maurice

\section{Echantillonnage et Définition des variables}

Notre échantillon porte de manière aléatoire sur 200 exploitations agricoles familiales à Maurice. Les données ont été collectées à l'aide d'un questionnaire réalisé par le logiciel Sphinx.

Les types de données collectées sont aussi bien des données quantitatives réalisées à l'aide de questionnaire destiné aux chefs d'exploitations agricoles familiales de la zone de l'étude. 
Ces données utilisées sont relativement la production agricole et les moyens de production agricole. Nous avons retenu pour cette étude 4 variables : 1 output et 3 inputs. Ils s'agissent de : kilogrammes

- production : il est mesuré par la production et exprimé en hectare ;

- superficies cultivées : les superficies cultivées sont exprimées en

- main d'œuvre : la main d'œuvre est approximée par la population active dans l'agriculture. Cet input est exprimé en homme/jour

- consommations intermédiaires : elles constituent la quantité d'engrais chimique ou organique appliqué, exprimée en kg par hectare.

\section{Présentation du modèle empirique}

Dans cette étude, l'efficacité technique des exploitations agricoles est mesurée par la méthode d'analyse par enveloppement de données (Data envelopment analysis-DEA). Il a été développé par (Charnes, et al., 1978) et est connu sous le nom de CCR Model (Farrell, 1957). Cette méthode est une approche non paramétrique basée sur l'utilisation des techniques de programmation linéaire pour mesurer l'efficacité et / ou l'inefficacité technique. Il construit une frontière par morceaux linéaire à partir des données observées, donc il ne nécessite aucune hypothèse sur la forme fonctionnelle et la répartition des termes d'erreur. Les ensembles de sortie et d'entrée définissent les frontières de possibilité de production contre lesquelles les performances techniques des activités de production peuvent être mesurées. Selon Coelli et al. (1998), il est nécessaire de sélectionner l'orientation à partir du modèle DEA orienté vers les entrées (inputs) ou du modèle DEA orienté vers les sorties (outputs). Coelli et al., (2002) suggèrent que le chef de l'exploitation doit en choisir un qui assure le contrôle des quantités (intrants et extrants). Comme les agriculteurs ont plus de contrôle sur les intrants que sur la production, nous utilisons un modèle de DEA axé sur les entrées. Il offre une plus grande flexibilité puisqu'il n'exige pas d'hypothèse a priori sur la relation fonctionnelle des intrants et des extrants.

La méthode DEA calcule les scores d'efficacité technique des différentes exploitations agricoles à partir d'une frontière d'efficacité. Les exploitations agricoles localisées sur la frontière sont considérées comme techniquement efficace avec un score de $1(100 \%)$ et celles localisées sous la frontière sont inefficace avec un score inférieur à 1 (Coelli et al., 2002; Heidari et al., 2011). Ces exploitations agricoles inefficaces disposent donc d'une marge d'amélioration de leur performance (Cooper, 2006). Elles pourront se référer aux exploitations agricoles techniquement efficaces pour appliquer leurs meilleures pratiques. 
Ce modèle a été initialement proposé par Charnes (1978) et est construit comme suit :

$$
\begin{aligned}
& \phi=\frac{\sum_{n}^{N}=1 \mu_{n} y_{n . j}}{\sum_{p}^{P} 1 v_{p} x_{p . j}} \\
& T E\left(x_{i}, y_{i}\right)=\min \phi\left(\phi_{j}, x_{i}, y_{i}\right)
\end{aligned}
$$

Pour :

$\sum_{j=1}^{J} \beta_{j} y_{n . j} \geq y_{n .0}$

$\sum_{j=1}^{J} \beta_{j} x_{p} \geq \phi \cdot x_{p .0}$

$\sum_{j=1}^{J} \beta_{j}=1$

$\beta_{j} \geq 0$

$\phi$ étant l'estimation de l'efficacité technique à calculer pour chaque exploitation ;

y les inputs (entrées) utilisées et $\mu$ la quantité des entrées ;

$\mathrm{x}$ les outputs (sorties) obtenues et $v$ la quantité des sorties ;

$\beta$ le vecteur d'intensité déterminant l'efficacité technique pour chaque exploitation ;

$\mathrm{n}$ est le nombre de sorties $(\mathrm{n}=1,2, . ., \mathrm{N})$;

$p$ est le nombre d'entrées $(\mathrm{p}=1,2, \ldots, \mathrm{P})$;

j représente le jème DMU (unité de prise de décision).

Les hypothèses sous-jacentes de ce modèle sont que l'exploitation agricole $\mathrm{j}(1,2, \ldots, \mathrm{J})$ produit la sortie $\mathrm{y}_{\mathrm{n}}$ en utilisant une combinaison d'intrants $\mathrm{x}_{\mathrm{p}}$ (main-d'œuvre, la terre, engrais); et une frontière de production axée sur les entrées des rendements d'échelle variable (VRS). La fonction objective $\phi_{j}$ est un scalaire qui représente le niveau minimum auquel l'utilisation des entrées peut être réduite sans altérer le niveau de sortie. Une exploitation est considéré comme techniquement efficace si $\phi_{j}=1$, alors qu'une exploitation avec $\phi_{j}<1$ est considéré comme techniquement inefficace. Un $\phi_{j}$ index implique que l'utilisation de toutes les entrées ne peut pas être réduite en même temps, bien qu'une variation de l'utilisation de l'une d'entre elles puisse améliorer l'efficacité (Iraizoz, et al., 2003). 
Dans la deuxième étape, le modèle Tobit a été utilisé pour identifier les facteurs associés à l'efficacité. Le modèle Tobit est couramment utilisé pour régresser les estimations de l'efficacité technique par rapport aux facteurs susceptibles d'influencer l'efficacité technique.

L'équation suivante a été estimée, sur la base des données primaires obtenues :

$T E=\beta_{1}+\beta_{2} A g+\beta_{3} E d u c+\beta_{4} S u p+\beta_{5} A c t+\beta_{6} E n g+\beta_{7} A d h+$ $\beta_{8} A u t S+\varepsilon(3)$

TE les scores d'efficacités obtenus par le DEA. Par conséquent, chaque DMU a un coefficient d'efficacité positif, limité à l'intervalle 0 à 1 ; $A g$ l'âge du producteur (en années); Educ le niveau de scolarité du producteur (en années); Sup la superficie cultivée (en hectares); Act le nombre d'actifs de l'exploitation; Eng les quantités d'engrais chimique et organique utilisées, Adh l'adhésion du chef d'exploitation à une organisation de producteurs ; AutS les sources de revenu non agricole ; $\varepsilon$ le terme d'erreur.

\section{Résultats et discussion}

\section{Analyse de l'efficience technique des exploitations familiales à Maurice}

L'essentiel de la production agricole concerne : la canne à sucre, le thé, le tabac et les cultures vivrières (les haricots et pois, les patates, le maïs, l'arachide, l'oignon la tomate, les fruits et les légumes). La superficie cultivée sous canne à sucre est passée de 57081 hectares en 2014 à 56872 ha en 2015 entrainant ainsi une baisse de $0,9 \%$ de la production. La production de thé vert a décru de $11,5 \%$ suite à une diminution des surfaces cultivées de $14,6 \%$ entre 204 et 2015. La superficie des cultures vivrières a aussi baissée de 4,5\%, impliquant une production qui est passée de 113,957 tonnes en 2014 à 102,663 tonnes en 2015(Statistics Mauritius, 2016).

La région est très propice à l'agriculture, mais le développement de ce dernier a été entravé par le climat sec et le manque d'installations d'irrigation. Maurice, est aussi marqué par le plus haut taux d'abandon des terres agricoles ou sa conversion à d'autres fins notamment au Nord (ADB, 2004).

Les résultats du tableau $N^{o} 1$ montrent que la quantité moyenne de la production agricole est de $8457,57 \mathrm{~kg}$ pour une superficie cultivée de 0,64 ha allant de 0,1 à 7 hectares. La main d'œuvre utilisée représente 2,05 hommes/jour et la quantité moyenne d'engrais utilisé est de 105,09 kg, avec une consommation moyenne d'engrais variant de $50 \mathrm{~kg}$ à $950 \mathrm{~kg}$. En effet, la taille moyenne des ménages agricoles est de 3,8 personnes (Statistics Mauritius, 2014). Ils peuvent être catégorisés en petit exploitant (moins de 0,42 hectares), moyen exploitant (de 0,42 à 5 hectares), à gros exploitant (plus de 5 hectares) rapporté par Ramasawmy et Fort, (2010). La majorité de ces 
producteurs produisent en plein champ, et leurs exploitations sont très peu mécanisées.

Tableau 1 - Statistiques descriptives des variables utilisés dans le modèle e

\begin{tabular}{|c|c|c|c|c|c|}
\hline Variables & Obs & Mean & Std. Dev. & Min & Max \\
\hline Production agricole (kg) & 200 & 8457.57 & 24606.91 & 0 & 280000 \\
\hline Nombre d'Actifs (homme-jour) & 200 & 2.051852 & 0.9272297 & 1 & 4 \\
\hline Superficie cultivée (ha) & 200 & 0.6438889 & 0.8509662 & 0,1 & 7 \\
\hline Quantité d'engrais (kg) & 200 & 105.09 & 6.561274 & 50 & 950 \\
\hline
\end{tabular}

Le Tableau $N^{0} 2$ montre la distribution du nombre d'exploitations agricoles et les statistiques récapitulatives pour les scores d'efficacité technique en termes de rendements d'échelle variables (VRS), de rendements d'échelle constants (CRS) et d'efficacité d'échelle (SE). L'efficacité technique moyenne dans le cadre du rendement d'échelle variable est de 0,726 , ce qui signifie qu'en moyenne, les répondants ont pu obtenir environ $72,6 \%$ de la production potentielle d'une combinaison donnée d'intrants. Cela implique également qu'environ $27,4 \%$ de la production est abandonnée en raison d'une inefficacité technique. En d'autres termes, l'insuffisance de la production observée de la production à la frontière reflétait principalement l'utilisation inefficace des facteurs qui étaient sous le contrôle des agriculteurs. Les niveaux d'efficacité technique des exploitations variaient de 0,25 à 1 . Cela signifie qu'il est possible d'augmenter la production agricole de $27,4 \%$ par rapport au niveau actuel des intrants.

Les résultats montrent que 46,5\% de l'échantillon sont techniquement efficaces sous rendement d'échelle variable. Pour être plus efficace, ils doivent diminuer en moyenne de $27,4 \%$ de leur consommation d'inputs.

Tableau 2. Répartition du nombre d'exploitations agricoles selon le score d'efficience

\begin{tabular}{|c|c|c|c|}
\hline & \multicolumn{2}{|c|}{ Maurice } \\
\hline Score d'efficience & vrste & crste & scale \\
\hline $\mathbf{0 . 0 0 - 0 . 2 5}$ & 10 & 171 & 160 \\
\hline $\mathbf{0 . 2 5}-\mathbf{0 . 5 0}$ & 25 & 15 & 16 \\
\hline $\mathbf{0 . 5 0 - 0 . 7 5}$ & 62 & 8 & 10 \\
\hline $\mathbf{0 . 7 5 - 0 . 9 9}$ & 10 & 1 & 4 \\
\hline $\mathbf{1 . 0 0}$ & 93 & 4 & 200 \\
\hline Total & 200 & 200 & 0,002 \\
\hline Min & 0,25 & 0,002 & 1 \\
\hline Max & 1 & 1 & 0,163 \\
\hline Moyenne & 0,726 & 0,117 & \\
\hline
\end{tabular}

Notes: crste $=$ Efficience technique vers CRS

vrste $=$ Efficience technique vers VRS scale $=$ Efficience d'échelle $(\mathrm{SE})$

\section{Analyse de déterminants de l'efficience technique}

Dans la première étape de l'analyse, l'efficacité technique des exploitations individuelles a été estimée par la DEA. Comme la frontière de production dans l'approche DEA est déterministe, les efficacités résultantes 
incluent les données. Par conséquent, dans la deuxième étape de la présente analyse, les déterminants de l'inefficacité ont été calculés en utilisant le modèle de régression Tobit. Les résultats estimés sont présentés dans le tableau $N^{0} 4$.

L'âge du chef de ménage est positivement lié à l'efficacité agricole, mais la relation n'était pas significative. En effet, les agriculteurs âgés étaient techniquement moins efficaces que leurs homologues plus jeunes. Cela implique que les agriculteurs âgés étaient techniquement moins efficaces que leurs homologues plus jeunes. Cela pourrait être attribué en partie à des facteurs psychologiques (attachement aux méthodes traditionnelles d'agriculture) et en partie à des facteurs économiques (les agriculteurs âgés sont généralement à risque). Des conclusions similaires ont été trouvées par Sibiko et al., (2012).

Le sexe féminin du chef de famille a un effet positif sur l'efficacité technique des exploitations, et la relation est significative au seuil de $1 \%$. Les résultats suggèrent que les chefs d'exploitation femme augmentent le niveau de probabilité d'efficacité technique par rapport à leurs homologues hommes. Cela veut dire que les agriculteurs de sexe féminin sont plus efficaces sur le plan technique que ceux de sexe masculin.

Le nombre d'actif de l'exploitation n'est pas significatif et a un effet négatif sur l'efficacité technique. La productivité marginale du travail est positive mais l'insuffisance de la main-d'œuvre agricole est un des contraintes majeures de l'agriculteur mauricien. Le résultat suggère que les stratégies qui tiennent compte d'une augmentation de la main-d'œuvre agricole devraient être mises en place (Tableau 4).

Les agriculteurs plus éduqués étaient susceptibles d'être plus efficaces que leurs homologues moins instruits. Les raisons plausibles d'une corrélation positive pourraient être leurs meilleures compétences, l'accès à l'information et une bonne planification agricole. Cela aurait naturellement pu aider les personnes interrogées à prendre de meilleures décisions techniques et à leur permettre d'allouer les intrants de manière efficace et efficiente. Comme l'on montré les travaux de Coelli et Battese (1996) et Bravo-Ureta, et al., (1997). Cependant, les résultats montrent que le niveau d'éducation du chef d'exploitation n'est pas significatif et que l'éducation primaire est négativement corrélé avec l'efficacité technique des répondants.

La superficie cultivée s'est révélée relativement liée à l'efficacité des exploitations, et la relation fortement significative (1\%). Le fait que l'efficacité technique soit positivement et significativement liée à la superficie suggère qu'il existe une marge pour augmenter la productivité de l'exploitation. Les résultats calculés ont suggéré qu'une augmentation de la superficie augmentera l'efficacité de l'exploitation. Ceux-ci ont peut-être aidé les répondants à prendre de meilleures décisions techniques et d'augmenter efficacement leurs superficies. 
La principale occupation de l'agriculteur a eu un effet négatif sur l'efficacité technique de l'exploitation. Les résultats estimés suggéraient que dès que la structure professionnelle passait de l'agriculture à d'autres professions, le niveau de probabilité de l'efficacité technique diminuait. Les agriculteurs dont l'activité principale reste l'agriculture sont donc plus efficaces que ceux qui exercent un emploi ou des entreprises ou toute autre activité génératrice de revenus. Cependant, nos résultats montrent que le commerce et à un emploi (salaire) sont significatifs aux seuils de 5\% et 1\% respectivement et sont positivement liée à l'efficacité technique de la productivité agricole. Cela signifie que ces professions génèrent un revenu disponible supplémentaire assuré et régulier les permettant de financer leurs activités agricoles.

Cela pourrait être lié à l'hypothèse que dans l'économie du travail, le salaire, qui améliore les dépenses de consommation (ou le revenu), entraîne une meilleure nutrition des travailleurs et donc une amélioration de l'efficacité technique de la production.

Tableau 4 : Facteurs associés à l'efficacité technique

\begin{tabular}{|c|c|c|c|c|c|c|}
\hline Variables & Coef. & Std. Err. & $\mathrm{t}$ & \multicolumn{2}{|c|}{$\mathrm{P}>\mathrm{t}$} & \multicolumn{2}{|c|}{ [95\% Conf.Interval } \\
\hline Sexe & & & & & & \\
\hline Féminin & 17127.1 & 5278.488 & 3.24 & $0.001^{* * *}$ & 6731.729 & 27522.48 \\
\hline Age & 107.683 & 146.9515 & 0.73 & 0.464 & -181.7211 & 397.0871 \\
\hline Nombre d'actifs & -900.4776 & 1661.396 & -0.54 & 0.588 & -4172.406 & 2371.451 \\
\hline Education & & & & & & \\
\hline Illettré & 10319.6 & 7339.74 & 1.41 & 0.161 & -4135.176 & 24774.37 \\
\hline Primaire & -1747.43 & 3443.063 & -0.51 & 0.612 & -8528.147 & 5033.287 \\
\hline Université & 3081.335 & 5858.816 & 0.53 & 0.599 & -8456.929 & 14619.6 \\
\hline Superficie & 14775.67 & 1711.216 & 8.63 & $0.000^{* * *}$ & 11405.63 & 18145.71 \\
\hline Régime foncier & & & & & & \\
\hline Location & -4390.285 & 2912.403 & -1.51 & 0.133 & -10125.93 & 1345.357 \\
\hline Location/propriétaire & -961.8903 & 6527.077 & -0.15 & 0.883 & -13816.22 & 11892.44 \\
\hline Fertilisant & & & & & & \\
\hline Aucun & 5071.117 & 15622.19 & 0.32 & 0.746 & -25694.99 & 35837.22 \\
\hline Compost & -17091.51 & 8474.349 & -2.02 & $0.045 * *$ & -33780.77 & -402.2602 \\
\hline Organique & -4847.752 & 6665.788 & -0.73 & 0.468 & -17975.25 & 8279.749 \\
\hline Revenu non-agricole & & & & & & \\
\hline Salaire & 11426.93 & 3573.08 & 3.20 & $0.002^{* * *}$ & 4390.158 & 18463.7 \\
\hline Commerce & 13203 & 5944.299 & 2.22 & $0.027 * *$ & 1496.389 & 24909.61 \\
\hline Embouche & 8846.164 & 6410.229 & 1.38 & 0.169 & -3778.045 & 21470.37 \\
\hline Autres & 9089.839 & 5497.936 & 1.65 & 0.100 & -1737.713 & 19917.39 \\
\hline Adhésion Organisation & & & & & & \\
\hline oui & 4905.429 & 3732.479 & 1.31 & 0.190 & -2445.257 & 12256.12 \\
\hline cons & -12429.88 & 8677.903 & -1.43 & 0.153 & -29520.01 & 4660.245 \\
\hline /sigma & 21619.23 & 985.7962 & & & & \\
\hline Niveaux de significativité $: *$ & $: 10 \% * *: 5 \% * * *: 1 \%$ & 19677.82 & 23560.64 \\
\hline
\end{tabular}




\section{Conclusion}

Cet article a estimé l'efficacité technique de la production agricole des exploitations familiales à Maurice et a établi les facteurs qui influent sur l'efficacité technique. Nous avons utilisé la méthode en deux étapes qui consiste à coupler un modèle DEA pour estimer l'efficacité technique à un modèle de régression Tobit pour établir les facteurs qui influent sur l'efficacité technique. Les résultats empiriques fournissent des preuves que l'inefficacité technique dans la production agricole existe parmi les répondants. L'efficacité technique moyenne est estimée à 72,6\%. Ce qui implique que les exploitations agricoles familiales peuvent réduire l'utilisation d'intrants de $27,4 \%$ et produire encore plus que le niveau de production actuel.

L'analyse du modèle de régression Tobit indique que le sexe, la taille de la superficie cultivée et le salaire influent de manière significative sur l'efficacité technique de la production agricole. En effet, les agriculteurs ayant les plus grandes surfaces cultivées sont plus efficaces sur le plan technique que ceux de petites surfaces. Les femmes agricultrices sont techniquement plus efficaces que les hommes dans leur exploitation. De plus, le travail salarié augmente l'efficacité technique.

Les interventions politiques devront donc être axées sur l'accès à la terre, l'amélioration du niveau d'éducation formelle des producteurs, la disponibilité des intrants et le renforcement des organisations paysannes afin d'accroître l'efficacité technique globale des producteurs à Maurice.

\section{References:}

1. Abatania, L., Hailu, A., et Mugera, A. (2012). Analysis of farm household technical efficiency in Northern Ghana using bootstrap DEA. The 56th annual conference of the Australian Agricultural and Resource Economics Society, 7-10 February, 33p.

2. ADB. (2004). Northern plains irrigation project. Appraisal report, Agriculture and rural development department, Port-Louis: Republic of Mauritius, 58p.

3. Alene, A., Manyong, V., et Gockowski, J. (2006). The production efficiency of intercropping annual and perennial crops in southern Ethiopia: A comparison of distance functions and production frontiers. Agricultural Systems, 91(1-2), 51-70.

4. Banque Mondiale. (2014). Agriculture, valeur ajoutée (\% du PIB). [En ligne] Available at: $\underline{\text { http://donnees.banquemondiale.org }}$ [Accès le 22 Avril 2015].

5. Bhatt, M. S., et Bhat, S. A. (2014). Technical efficiency and farm size productivity - micro level evidence from jammu \& kashmir. International Journal of Food and Agricultural Economics, 2(4), 2749. 
6. Bravo-Ureta, B. et al., (1997). Technical efficiency in farming: A meta-regression analysis. Journal of Productivity Analysis, Volume 27, p. 57-72.

7. Charnes, A., Cooper, W., et Rhodes , E. (1978). Measuring the efficiency of decision making units. European Journal of Operational Research (2), 429-441.

8. Chavas, J., Petrie, R., et Roth, M. (2005). Farm Household Production Efficiency: Evidence from the Gambia. American Journal of Agricultural Economics, 87(1), 160-179.

9. Chiona, S. (2011). Technical and Allocative Efficiency of Smallholder Maize Farmers in Zambia. Unpublished MSc Thesis, University of Zambia, Lusaka, Zambia, 66p.

10. Clemente, F., Lírio, V. S., et Gomes, M. M. (2015). Technical efficiency in Brazilian citrus production. Bio-based and Applied Economics, 4(2), 165-178.

11. Coelli , T., Rao, D., et Batese, G. (1998). An introduction to efficiency and productivity analysis. Kluwer Academic Publishers, Boston, 2139

12. Coelli, T. J. et Battese, G. E., 1996. Identification of factors which influence the technical inefficiency of Indian farmers. Australian Journal of Agricultural Economics, 40,103-128.

13. Coelli, T., Rahman, S., et Thirtle, C. (2002). Technical, Allocative, Cost and Scale Efficiencies in Bangladesh Rice Cultivation: A Nonparametric Approach. Journal of Agricultural Economics, 53(3), 607626.

14. Cooper, W., Seiford, L., et Tone, K. (2006). Introduction to Data Envelopment Analysis and its uses. Springer Science and Business, New York, 354p

15. Dhehibi, B., Alimari, A., Haddad, N., et Aw-Hassan, A. (2014). Technical Efficiency and Its Determinants in Food Crop Production: A Case Study of Farms in West Bank, Palestine. Journal of Agricultural Science and Technology, 16, 717-730.

16. Diogo, R. V. C., Agandan , E. M. M., Nouatin , G. S. et Djedje, M., (2017). Modes de gestion de la fertilité des sols des agro-éleveurs peuls au Nord-Ouest Bénin : implications pour la sécurité alimentaire. Annales de l'Université de Parakou, Sciences Naturelles et Agronomie, 1, 74-81.

17. FAO. (2005). Aquastat Mauritius, water report. [En ligne] Available at: http://www.fao.org/nr/water/aquastat/countries_regions/MUS/MUSCP_eng.pdf [Accès le 15 Aout 2015]. 
18. Farrell, M. (1957). The Measurement of productive efficiency. Journal of the Royal Statistical Society, 120, 253-281.

19. Heidari , M., Omid , M., et Akram , A. (2011). Using Nonparametric Analysis (DEA) for Measuring Technical Effi ciency in Poultry Farms. Brazilian Journal of Poultry Science, 13(4), 271-277.

20. HRDC. (2012). A Study on Labour Shortage in the Agricultural Sector in Mauritius. Port-Louis : Human Resource Development council, $104 \mathrm{p}$.

21. IEDOM. (2009). Le développement économique de Singapour et de l'Ile Maurice: Eléments de réflexion pour un développement durable aux Antilles françaises et à La Réunion. Les Notes de 1'Institut d'émission, Paris: Institut d'Emission des Départements d'Outre-Mer, $52 \mathrm{p}$.

22. IFAD. (2013). Enabling poor rural people to overcome poverty in Mauritius. Antananarivo, Madagascar: International Fund for Agricultural Development (IFAD), 4p.

23. Iraizoz, B., Rapun, M., et Zabaleta, I. (2003). Assessing the technical efficiency of horticultural production in Navara. Agricultural Systems, 78, 387-403.

24. Javed, M., Adil, S., Ali, A., \& Raza, M. (2010). Measurement of Technical Efficiency of Rice - Wheat System in Punjab, Pakistan. Journal of Agricultural Resource, 48(2), 227-238.

25. Kamminga, A.T. (2008). Vulnerability assessment of potential soil erosion; a case study on Mauritius,. Master thesis BSc., Faculty of Science (FNWI), University of Amsterdam, 63p.

26. Le Roux, Jacobus Johannes. (2005). Soil erosion prediction under changing land use on Mauritius, Master of Science Geography, University of Pretoria, 180p.

27. MAIF. (2008). A sustainable Sustainable Diversified Agri-food Sector Strategy For Mauritius 2008 - 2015. Port-Louis, Mauritius: Ministry of Agro-Industries and Fisheries , 43p.

28. MAIFS. (2013). Food Security Fund: Strategic Plan -2013-2015. PortLouis: Ministry of Agro Industry and Food Security of Mauritius, 52p.

29. Mardamootoo, T. (2009). Evaluation of the phosphorus status of sugarcane soils in Mauritius using agronomic and environmental criteria. Dissertation for the Magister Scientiae degree in Soil Science in the Department of Soil, Crop and Climate, Faculty of Natural and Agricultural Sciences, Bloemfontein, South Africa: University of Free State, 143p.

30. Osawe, O., Akinyosoye, V., et Omonona, B. (2008). Technical efficiency of small scale farmers: an application of the stochastic frontier production function to fish farmers in Ibadan metropolis, Oyo 
State, Nigeria. Journal of Economics and Rural Development, 16(1), 71-82.

31. Padilla-Fernandez, et Nuthall, P. (2009). echnical Efficiency in the Production of Sugarcane in Central Negros Area, Philippines. An Application of DEA. Journal of International Society for Southeast Asian Agricultural Sciences, 15(1), 77-90.

32. Rahman, S., et Umar, H. (2009). Measurement of technical efficiency and its determinants in crop production in Lafia local Government area of Nasarawa State, Nigeria. Journal of Tropical Agriculture, Food, Environment and Extension, 8(2), 90 - 96.

33. Ramasawmy et Fort. (2010). Effets d'un changement institutionnel, la réforme du protocole sucre (ACP-UE), sur la filière légumes frais à l'île Maurice. The 116th EAAE Seminar "spatial dynamics in agrifood systems: implications for sustainability and consumer welfare"?, 27th30th October, $17 \mathrm{p}$.

34. Rios, A., \& Shively, G. (2005). Farm size and nonparametric efficiency measurements for coffee farms in Vietnam. Selected Paper prepared for presentation at the American Agricultural Economics Association, Meeting, Providence, Rhode Island, July 24-27, 22p.

35. Sibiko, K. W. et al., (2012). Analysis of determinants of productivity and technical efficiency among smallholder common bean farmers in eastern Uganda. Current Research Journal of Economic Theory, Volume 53, 44-55.

36. Statistics Mauritius. (2014). Digest of agricultural statictics-2013, Ministry of Finance and Economic Development, 145p.

37. Statistics Mauritius. (2016). Digest of agricultural statictics -2015, Ministry of Finance and Economic Development, 151p.

38. Statistics Mauritius. (2014). Census of Agriculture - Main Results, Ministry of Finance and Economic Development, 22p

39. UNFCCC. (2005). Climate change, small island developing States, climate change secretariat, Bonn, Germany, 32p.

40. UNFCCC. (2010). Second National Communication of the Republic of Mauritius. Mauritius Meteorological Services, Ministry of Environment and Sustainable Development, Vacoas, Mauritius: Government of Mauritius, 148p.

41. USAID. (2015). Mauritius 2015. [En ligne] Available at: https://idea.usaid.gov/prepared/FactSheets/mauritius.pdf [Accès le 10 Février 2016].

42. Yusuf, S., et Malomo, D. (2009). Technical Efficiency of Poultry Egg Production in Ogun State: A Data Envelopment Analysis Approach. International Journal of Poultry Science, 6(9), 622-629. 